wicklung bis zur Fabrikationsreife war Hr. Dr. Ernst D o n a t maßgeblich beteiligt; auch an dieser Stelle möchte Verf. ihm Dank für die Unterstützung sagen. Die Fabrikationsreife der Bleiglanz-Netzzelle war im Sommer 1936 erreicht.

Mit intermittierender Bestrahlung bereits 1934/35 bis zu etwa $10000 \mathrm{~Hz}$ durchgeführte Messungen ließen im Frequenzgang des lichtelektrischen Effektes von Bleiglanz keinerlei Spannungsabfall bei höheren Frequenzen, also keine Trägheit erkennen. Die Verwendung der BleiglanzNetzzelle für Fernsehbildzerleger, auch und gerade mit Bildabtastung durch Infrarot von etwa $1,5 . \mu$ bis zu etwa $3,5 \mu$, lag daher nahe 7 , setzte allerdings — auch bei mechanischer Bildzerlegung - eine wesentlich weitergehende Frequenzunabhängigkeit, bis zu etwa $10^{5} \mathrm{~Hz}$, voraus. Letzteres konnte wegen dringender andersartiger Aufgaben vom Verf. experimentell nicht restlos weiterverfolgt werden, war aber in Hinsicht auf den Frequenzgang bis zu $10000 \mathrm{~Hz}$ als wahrscheinlich angenommen. Um so erfreulicher ist die immerhin teilweise Bestätigung

7 Patentanmeldg. S. 119605 VIII a/21 a ${ }^{1}$ der Dr. Ge org Seibt AG. vom 31. August 1935, geheim erklärt am 24. Oktober 1935, Erfinder: Fritz Michelssen.

8 Patentanmeldg. S. 115613 VIII c/21 g der Dr. Georg S eibt AG. vom 5. Oktober 1934, geheim erklärt am 9. Juli 1935, Erfinder: Fritz Michelssen. seiner Vermutung durch die von $\mathrm{K}$ ar olus und Mangold (vgl. voranstehenden Aufsatz) festgestellte Tatsache, daß die Bleiglanznetzzellen bis $\mathrm{zu} \sim 40000 \mathrm{~Hz}$ keine Frequenzabhängigkeit aufweisen.

Die Bedeutung des negativen Temperaturkoeffizienten des lichtelektrischen Effektes an sardinischem Bleiglanz (Vakuumanordnung) (vgl. l. c. 5, S. 132) stellte Verf. bereits im September 1934 fest und schlug daraus folgend auch schon eine besondere Kühlvorrichtung für BleiglanzPhotozellen im Vakuum vor ${ }^{8}$.

Über die technische Anfangsentwicklung der Bleiglanz-, $\mathrm{PbS}-, \mathrm{PbSe}-$ und PbTe-Infrarot-Photozellen und deren Anwendung, z. B. für Nachrichten- und Ortungszwecke, für Temperaturmessungen, Tonfilm usw. mit sehr vielen weiteren Einzelheiten soll an anderer Stelle berichtet werden $\mathbf{9}$.

9 Verf. hat ab 1938 in dieses von ihm selbständig begonnene Arbeitsgebiet keinen Einblick mehr gehabt, auch sind ihm die seit 1946 von anderer Seite gelegentlich zitierten „Forschungsarbeiten über infrarote Strahlungsempfänger, herausgegeben vom OKH/Wa. F., Berlin 1944“ nicht bekannt; sie sind gemäß der von den Zentralkatalogen in Berlin und Köln gegebenen Auskünfte auch heute in keiner deutschen öffentlichen Bibliothek einzusehen oder zu entleihen, nicht einmal in den Sperrbüchereien vorhanden.

\title{
NOTIZEN
}

\section{Bestimmung der Massenzahl der 3,9-min-Aktivität des Selens}

Von A. F l a m mers feld und W. Her r

Max-Planck-Institut für Chemie, Mainz

(Z. Naturforschg. 5 a, 569-570 [1950]; eingeg. am 6. Okt. 1950)

Bei der Bestrahlung von Selen mit langsamen und schnellen Neutronen wurde kürzlich eine neue SelenAktivität von $T=3,9$ min Halbwertszeit aufgefunden, die sowohl durch $(\mathrm{n}, \gamma)$ - wie durch $(\mathrm{n}, 2 \mathrm{n})$-Prozesse aus den stabilen Selen-Isotopen erzeugt werden kann und die als Isomer eines der Kerne ${ }^{77} \mathrm{Se},{ }^{79} \mathrm{Se}$ oder ${ }^{81} \mathrm{Se}$ gedeutet wurde, wobei über die Massenzahl keine eindeutigen Aussagen möglich waren 1 .

Zur genaueren Festlegung der Massenzahl wurden Versuche unternommen, die 3,9-min-Aktivität durch (n,p)Prozeß aus Brom darzustellen. Zu diesem Zwecke wurden 5 bis $10 \mathrm{ccm}$ einer 40-proz. Bromwasserstoffsäure mit $\mathrm{Li}+\mathrm{D}$ - und $\mathrm{Be}+\mathrm{D}-\mathrm{Neutronen}$ der Hochspannungsanlage unseres Instituts $\left(E_{\mathrm{D}}=1,4 \mathrm{MeV}\right)$ bestrahlt. $\mathrm{Zu}$ dieser aktiven $\mathrm{HBr}$-Lösung wurden $1,2 \mathrm{mg}$ inaktives Selen $\left(\mathrm{SeO}_{2}\right)$ zugegeben und das Ganze in eine siedende Lösung von $200 \mathrm{mg}$ Hydrazinsulfat in $15 \mathrm{ccm}$ Wasser geschüttet. Nach $30 \mathrm{sec}$ wurde der Niederschlag von elementarem Selen abfiltriert, gut mit heißer konz. Bromwasserstoffsäure und anschließend mit Wasser gewaschen, trocken gesaugt und gemessen. Vorversuche mit dem 57 -min-Selen als Indikator hatten sichergestellt, daß unter diesen Bedingungen eine nahezu quantitative Fällung des vier- und sechswertigen Sélens erfolgt. Als Filter erwiesen sich Membranfilter („2 sec“) als sehr geeignet. Für die chemische Abtrennung waren 1,5 min notwendig; die Bestrahlungszeit betrug gewöhnlich $3 \mathrm{~min}$, die Messung der Se-Aktivitäten geschah in einem Zählrohr mit dünnem Glimmerfenster $\left(3 \mathrm{mg} / \mathrm{cm}^{2}\right)$, um den gesuchten $3,9-$ min-Körper trotz seiner sehr weichen Strahlung nachweisen zu können.

Als Ergebnis eines solchen Versuches mit $\mathrm{Li}+\mathrm{D}-\mathrm{Neu}-$ tronen zeigen sich in der Se-Fraktion nur 2 Halbwertszeiten von $T=57 \mathrm{~min}$ und $T=3,9 \pm 0,1 \mathrm{~min}$. Zur Untersuchung der Energien der Strahlungen beider Körper wurden noch Versuche ausgeführt, bei denen zusätzliche Absorptionsfolien von 20 bis $100 \mu$ Aluminium zwischen Präparat und Zählrohr eingeschaltet wurden. Dabei ergab sich wieder, daß die 3,9-min-Aktivität außerordentlich weiche Strahlung aussendet und z. B. durch eine zusätzliche $50 \mu$ Aluminium-Absorberfolie bereits ganz absorbiert wird. Die so ermittelte Reichweite stimmt überein mit der früher gemessenen von $9,5 \mathrm{mg} / \mathrm{cm}^{2} \mathrm{Al}^{1}$, so daß die Identität des hier aus $\operatorname{Br}(n, p)$ entstandenen 3,9-min-Körpers mit dem früher aus $\operatorname{Se}(n, \gamma)$ und $\operatorname{Se}(n, 2 n)$ gefundenen Körpers gleicher Halbwertszeit nicht bezweifelt werden kann.

Die 57-min-Aktivität ist zweifellos ${ }^{81} \mathrm{Se}^{*}$, dessen Entstehung aus $\operatorname{Br}(n, p)$ bereits länger bekannt ist 2 . Bemer-

1 A. Fla m mersfeld u. Chr. Ythier, Z. Naturforschg. 5a, 401 [1950].

2 A.H. S nell, Physic. Rev. 52, 1007 [1937]; A. Langsdorf jr., u. E. Se grè, Physic. Rev. 57, 105 [1940]. 
kenswert ist, daß die Messungen mit Absorbern von mehr als $50 \mu$ vom Beginn an einen reinen 57-min-Abfall zeigen, ohne daß die 18-min-Halbwertszeit des zugehörigen Grundzustandes ${ }^{81} \mathrm{Se}$ bemerkbar wird. Trotzdem muß auch dieser Partner des Isomerenpaares direkt durch $\mathrm{Br}(\mathrm{n}, \mathrm{p})$ gebildet worden sein. Würde nämlich nur der 57-min-Körper allein auftreten, so müßte bei den kurzen Bestrahlungszeiten noch ein erheblicher 18-min-Anstieg infolge des sich nachbildenden, aus dem ${ }^{81} \mathrm{Se}^{*}$ entstehenden 81 Se erfolgen. Es wurde noch untersucht, ob das Intensitätsverhältnis der interessierenden Se-Aktivitäten bei Verwendung von $\mathrm{Be}+\mathrm{D}-\mathrm{Neutronen}$ das gleiche bleibt. Hierbei ergaben sich wieder alle 3 Se-Aktivitäten, aber in veränderter relativer Intensität. Das Verhältnis der auf unendlich lange Bestrahlung umgerechneten Anfangsaktivitäten ist hier rund $3,6: 1,59: 1$ für die 3,9-18-minbzw. 57-min-Perioden, während bei den $\mathrm{Li}+\mathrm{D}$-Versuchen die entsprechenden Verhältnisse 0,31:1,45:1 betragen.

Für die Zuordnung des 3,9-min-Selens ergeben sich also aus den vorliegenden Versuchen folgende Gesichtspunkte. Der Beweis der Entstehung aus mindestens einem der Bromisotope 79 und 81 durch (n,p)-Prozeß schließt die Massenzahl 77 aus und läßt die Wahl zwischen 79 und 81. Davon ist der Platz 81 bereits durch zwei Aktivitäten besetzt, so daß eine Zuordnung zu dieser Massenzahl die Annahme eines weiteren Isomers notwendig machen würde. Aus den Intensitäten beim Be+DVersuch kann man aber sofort folgern, daß weder der 18-min- noch der 57-min-Körper das Folgeprodukt des 3,9-min-Körpers sein kann, weil dann deren Intensität wesentlich größer sein müßte. Es besteht also kein genetischer Zusammenhang und eine Zuordnung zu 81 erforderte die Annahme einer vierten hypothetischen Aktivität großer Halbwertszeit und gleicher Massenzahl als Grundzustand zum 3,9-min-Körper. Diese Schwierigkeit wird vermieden, wenn der neuen Aktivität die Massenzahl des noch unbesetzten Platzes 79 zugeordnet wird. Für diese Zuordnung spricht weiter sehr stark die Betrachtung der Ausbeuten unter Berücksichtigung der Reaktionsenergien der (n,p)-Prozesse. Aus der $\beta$-Zerfallsenergie $(1,5 \mathrm{MeV})$ des ${ }^{81} \mathrm{Se}$ kann geschlossen werden, daß der Prozeß ${ }^{81} \mathrm{Br}(\mathrm{n}, \mathrm{p})^{81}$ Se mindestens $0,8 \mathrm{MeV}$ erfordert. Da der 57-min-Körper der Zustand höherer Energie ist, benötigt er noch etwas mehr Energie zur Bildung. Im Einklang mit einer sehr häufigen Beobachtung bei Isomeren wird daher bei den höheren $\mathrm{Li}+\mathrm{D}$-Neutronenenergien der 57-min-Körper relativ zum Grundzustand etwas häufiger gebildet als mit den niedrigeren $\mathrm{Be}+\mathrm{D}$ Neutronenenergien. Viel stärker aber ist noch die relative Bevorzugung des 3,9-min-Körpers bei Erniedrigung der Neutronenenergie, was darauf hindeutet, daß die Energietönung des betreffenden $\operatorname{Br}(n, p)$-Prozesses kleiner ist als die der beiden anderen. Nun ist ${ }^{79} \mathrm{Se}$ (Grundzustand) bisher noch unbekannt, weil es vermutlich sehr große Lebensdauer und daher kleine $\beta$-Zerfallsenergie besitzt. Somit kann erwartet werden, daß der Prozeß ${ }^{79} \mathrm{Br}(\mathrm{n}, \mathrm{p}){ }^{79} \mathrm{Se}$ weniger Energie erfordert als ${ }^{81 \mathrm{Br}(n, p)}{ }^{81} \mathrm{Se}$ im Einklang mit den obigen Ergebnissen.

Die geschilderten Tatsachen scheinen hinreichend, die Zuordnung der 3,9-min-Aktivität zur Massenzahl $79 \mathrm{zu}$ gestatten, so daß diese also als. ${ }^{79} \mathrm{Se}^{*} \mathrm{zu}$ betrachten ist.
Und zwar kann u. E. die Zuordnung denselben Grad von Zuverlässigkeit beanspruchen wie die der ${ }^{81} \mathrm{Se}-$ und ${ }^{81} \mathrm{Se}^{*}$ Aktivitäten, die zur Klasse B gehören.

\section{Massenabhängigkeit der Elektronenauslösung durch isotope Ionen \\ Von Werner Ploch \\ Physikalisches Institut der Universität Marburg \\ (Z. Naturforschg. 5 a, $570-571$ [1950]; eingeg. am 30. Sept. 1950)}

Die Abhängigkeit der Elektronenauslösung durch positive Ionen von der Isotopenmasse ist bisher nur im Falle der Wasserstoffisotope $\mathrm{H}_{2}$ und $\mathrm{D}_{2}$ ohne magnetische Trennung der Atom- und Molekülionen untersucht worden ${ }^{1}$. Nachdem neuerdings die Messung der Ionenströme im

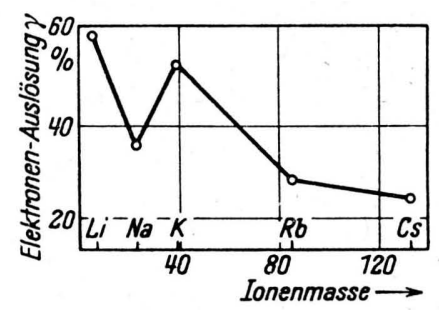

Abb. 1. Elektronen-Auslösung durch Alkali-Ionen von $1,2 \mathrm{keV}$ an nicht entgastem Molybdän. $\gamma=$ ausgelöster Elektronenstrom/einfallender Ionenstrom.

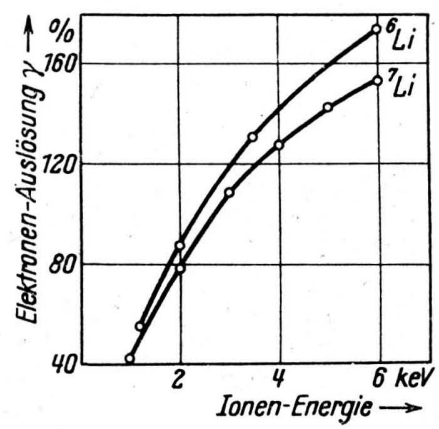

Abb. 2. Elektronen-Auslösung durch Lithium-Ionen an nicht entgastem Molybdän. $\gamma=$ ausgelöster Elektronenstrom/einfallender Ionenstrom.

Massenspektrometer mit Hilfe eines Elektronenvervielfachers zum Zwecke der Empfindlichkeitssteigerung Anwendung findet 2 , gewinnt die Kenntnis dieser Abhängigkeit und insbesondere ihr Zusammenhang mit der Art und Oberflächenbeschaffenheit des emittierenden Metalls sowie der Art und Energie der Ionen erneut Bedeutung.

Eine im Zusammenhang mit anderen massenspektrometrischen Untersuchungen durchgeführte Messung des Elektronenauslösungsfaktors $(\gamma=$ ausgelöster Elektronenstrom/einfallender Ionenstrom) lieferte folgende Ergeb-

1 Monica H e a le a, Physic. Rev. 55, 984 [1939] L.

2 W. T. Le la nd, Physic. Rev. 77, 634 [1950]. 